\title{
Some Properties on Estrada Index of Folded Hypercubes Networks
}

\author{
Jia-Bao Liu, ${ }^{1,2,3}$ Xiang-Feng Pan, ${ }^{1}$ and Jinde $\mathrm{Cao}^{2,4}$ \\ ${ }^{1}$ School of Mathematics Science, Anhui University, Hefei 230601, China \\ ${ }^{2}$ Department of Mathematics, Southeast University, Nanjing 210096, China \\ ${ }^{3}$ Anhui Xinhua University, Hefei 230088, China \\ ${ }^{4}$ Department of Mathematics, Faculty of Science, King Abdulaziz University, Jeddah 21589, Saudi Arabia
}

Correspondence should be addressed to Jinde Cao; jdcao@seu.edu.cn

Received 24 November 2013; Accepted 19 December 2013; Published 5 February 2014

Academic Editor: Qiankun Song

Copyright (C) 2014 Jia-Bao Liu et al. This is an open access article distributed under the Creative Commons Attribution License, which permits unrestricted use, distribution, and reproduction in any medium, provided the original work is properly cited.

Let $G$ be a simple graph with $n$ vertices and let $\lambda_{1}, \lambda_{2}, \ldots, \lambda_{n}$ be the eigenvalues of its adjacency matrix; the Estrada index $E E(G)$ of the graph $G$ is defined as the sum of the terms $e^{\lambda i}, i=1,2, \ldots, n$. The $n$-dimensional folded hypercube networks $F Q_{n}$ are an important and attractive variant of the $n$-dimensional hypercube networks $Q_{n}$, which are obtained from $Q_{n}$ by adding an edge between any pair of vertices complementary edges. In this paper, we establish the explicit formulae for calculating the Estrada index of the folded hypercubes networks $F Q_{n}$ by deducing the characteristic polynomial of the adjacency matrix in spectral graph theory. Moreover, some lower and upper bounds for the Estrada index of the folded hypercubes networks $F Q_{n}$ are proposed.

\section{Introduction}

Complex networks have become an important area of multidisciplinary research involving mathematics, physics, social sciences, biology, and other theoretical and applied sciences. It is well known that interconnection networks play an important role in parallel communication systems. An interconnection network is usually modelled by a connected graph $G=(V, E)$, where $V$ denotes the set of processors and $E$ denotes the set of communication links between processors in networks. Let $G$ be a graph with vertices labelled $1,2, \ldots, n$. The adjacency matrix $A(G)$ of $G$ is an $n \times n$ matrix with the $(i, j)$-entry equal to 1 if vertices $i$ and $j$ are adjacent and 0 otherwise. The spectrum of $G$ is the spectrum of its adjacency matrix and consists of the numbers $\lambda_{1} \geq \lambda_{2} \geq \cdots \geq$ $\lambda_{n}$. In this work we are concerned with finite undirected connected simple graphs (networks). For the underlying graph theoretical definitions and notations we follow [1].

The energy of the graph $G[2]$ is defined as

$$
E(G)=\sum_{i=1}^{n}\left|\lambda_{i}\right| .
$$

Another graph-spectrum-based invariant, recently put forward by Ernesto Estrada, is defined as

$$
E E=E E(G)=\sum_{i=1}^{n} e^{\lambda_{i}} .
$$

This graph invariant appeared for the first time in the year 2000, in a paper by Estrada [3], dealing with the folding of protein molecules. Estrada and Rodríguez-Velázquez showed that $E E$ provides a measure of the centrality of complex (communication, social, metabolic, etc.) networks $[4,5]$.

Denote by $M_{k}=M_{k}(G)=\sum_{i=1}^{n}\left(\lambda_{i}\right)^{k}$ the $k$ th spectral moment of the graph $G$. From the Taylor expansion of $e^{x}$, we have the following important relation between the Estrada index and the spectral moments of $G$ :

$$
E E(G)=\sum_{k=0}^{\infty} \frac{M_{k}(G)}{k !} .
$$

At this point one should recall [4] that $M_{k}(G)$ is equal to the number of self-returning walks of length $k$ of the graph 
$G$. The first few spectral moments of an $(n, m)$-graph with $m$ edges and $t$ triangles satisfy the following relations [4]:

$$
\begin{array}{cc}
M_{0}=\sum_{i=1}^{n}\left(\lambda_{i}\right)^{0}=n ; & M_{1}=\sum_{i=1}^{n}\left(\lambda_{i}\right)^{1}=0 ; \\
M_{2}=\sum_{i=1}^{n}\left(\lambda_{i}\right)^{2}=2 m ; & M_{3}=\sum_{i=1}^{n}\left(\lambda_{i}\right)^{3}=6 t .
\end{array}
$$

For $1 \leq i \leq n$, let $d_{i}$ be the degree of vertex $v_{i}$ in $G$. The first Zagreb index [6] of the graph $G$ is defined as $Z g(G)=\sum_{i=1}^{n} d_{i}^{2}$ :

$$
\begin{gathered}
M_{4}=\sum_{i=1}^{n}\left(\lambda_{i}\right)^{4}=2 Z g(G)-2 m+8 q ; \\
M_{5}=\sum_{i=1}^{n}\left(\lambda_{i}\right)^{5}=30 t+10 p+10 r
\end{gathered}
$$

where $p$ and $q$ are the numbers of pentagons and quadrangles in $G$, and $r$ is the number of subgraphs consisting of a triangle with a pendent vertex attached [7].

The hypercubes $Q_{n}$ is one of the most popular and efficient interconnection networks due to its many excellent performances for some practical applications. There is a large amount of literature on the properties of hypercubes networks [8-11]. As an important variant of $Q_{n}$, the folded hypercubes networks $F Q_{n}$, proposed by Amawy and Latifi [8], are the graphs obtained from $Q_{n}$ by adding an edge between any pair of vertices complementary addresses. The folded hypercubes $F Q_{n}$ obtained considerable attention due to its perfect properties, such as symmetry, regular structure, strong connectivity, small diameter, and many of its properties which have been explored [12-19].

The remainder of the present paper is organized as follows. In Section 2, we present some basic notations and some preliminaries in our discussion. The proofs of our main results are in Section 3 and some conclusions are given in Section 4 , respectively.

\section{Notations and Some Preliminaries}

In this section, we introduce some basic properties which will be used in the proofs of our main results.

Let $P_{F Q}(x)$ be the characteristic polynomial of the adjacency matrix of the folded hypercube $F Q_{n}$; the following results were shown in [12].

Lemma 1 (see [12]). The characteristic polynomial of the adjacency matrix of the $F Q_{n}(n \geq 3)$ is

$$
\begin{aligned}
P\left(F Q_{n} ; \lambda\right)= & {[\lambda-(n-7)][\lambda-(n-3)]^{3} P\left(F Q_{n-1} ; \lambda-1\right) } \\
& \times \prod_{i=2}^{n-2} P\left(F Q_{n-i} ; \lambda-(i-4)\right) .
\end{aligned}
$$

Lemma 2 (see [12]). For $F Q_{n}$ with $n \geq 3$, the spectrum of adjacency matrix is as follows:

(1) If $n \equiv 0(\bmod 2)$,

$$
\operatorname{Spec}\left(F Q_{n}\right)=\left(\begin{array}{ccccccc}
-n+1 & -n+5 & -n+9 & \cdots & n-7 & n-3 & n+1 \\
C_{n}^{0}+C_{n}^{1} & C_{n}^{2}+C_{n}^{3} & C_{n}^{4}+C_{n}^{5} & \cdots & C_{n}^{n-4}+C_{n}^{n-3} & C_{n}^{n-2}+C_{n}^{n-1} & C_{n}^{n}
\end{array}\right),
$$

(2) if $n \equiv 1(\bmod 2)$,

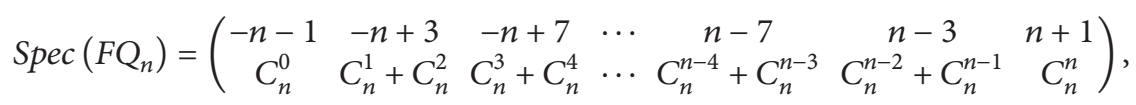

where $C_{n}^{i}$ are the binomial coefficients and the elements in the first and second rows are the eigenvalues of the adjacency matrix of $\mathrm{FQ}_{n}$ and the corresponding multiplicities, respectively.

Lemma 3 (see [20]). The eigenvalues of a bipartite graph satisfy the pairing property: $\lambda_{n-i+1}=\lambda_{i}, i=1,2, \ldots, n$. Therefore, if the graph $G$ is bipartite and if $\eta_{0}$ is nullity (the multiplicity of its eigenvalue zero), then

$$
E E(G)=\eta_{0}+2 \sum_{+} \cosh (i)
$$

where cosh stands for the hyperbolic cosine $\cosh (x)=$ $\left(e^{x}+e^{-x}\right) / 2$, whereas $\sum_{+}$denotes summation over all positive eigenvalues of the corresponding graph.

Lemma 4 (see [21]). Let $G$ be a graph with $m$ edges. For $k \geq 4$,

$$
M_{k+2} \geq M_{k}
$$

with equality for all even $k \geq 4$ if and only if $G$ consists of $m$ copies of $K_{2}$ and possibly isolated vertices and with equality for all odd $k \geq 5$ if and only if $G$ is a bipartite graph.

The following lemma is an immediate result of the previous lemma. 
Lemma 5 (see [22]). Let $G$ be an $(n, m)$ graph with $m$ edges. For $k \geq 4$,

$$
\sum_{i=1}^{n}\left(2 \lambda_{i}\right)^{k+2} \geq 4 \sum_{i=1}^{n}\left(2 \lambda_{i}\right)^{k}
$$

with equality for all even $k \geq 4$ if and only if $G$ consists of $m$ copies of $K_{2}$ and possibly isolated vertices and with equality for all odd $k \geq 5$ if and only if $G$ is a bipartite graph.

Lemma 6 (see [23]). Let $G$ be a regular graph of degree $r \neq 0$ and of order $n$. Then its Estrada index is bounded by

$$
e^{r}+(n-1) e^{-r /(n-1)} \leq E E(G)<n-2+e^{r}+e^{\sqrt{r(n-r)-1}} .
$$

Equality holds if and only if $\lambda_{2}=\lambda_{3}=\cdots=\lambda_{n}=-r /(n-1)$.

Lemma 7 (see [23]). The Estrada index $E E(G)$ and the graph energy $E(G)$ satisfy the following inequality:

$$
\frac{1}{2} E(G)(e-1)+n-n_{+} \leq E E(G) \leq n-1+e^{E(G) / 2},
$$

and equalities on both sides hold if and only if $E(G)=0$.

\section{Main Results}

3.1. The Estrada Index of Folded Hypercubes Networks $F Q_{n}$. In this section, we present some explicit formulae for calculating the Estrada index of $F Q_{n}$. For convenience, we assume that $C_{n}^{i}=0$ if $i<0$ or $i>n$.

Theorem 8. For any $F Q_{n}$ with $n \geq 3$, then

(1) $E E\left(F Q_{n}\right)=\sum_{i=0}^{n / 2}\left(C_{n}^{2 i}+C_{n}^{2 i+1}\right) e^{4 i-n+1}, i=0,1, \ldots, n / 2$, if $n \equiv 0(\bmod 2)$;

(2) $E E\left(F Q_{n}\right)=\sum_{i=0}^{n / 2}\left(C_{n}^{2 i-1}+C_{n}^{2 i}\right) e^{4 i-n-1}, i=0,1, \ldots,(n+$ $1) / 2$, if $n \equiv 1(\bmod 2)$,

where the $4 i-n+1$ and $4 i-n-1(i=0,1, \ldots, n / 2$ or $(n+$ 1)/2) are the eigenvalues of the adjacent matrix of $F Q_{n}$ and $C_{n}^{i}$ denotes the binomial coefficients.

Proof. By Lemma 1, the characteristic polynomial of the adjacent matrix of $F Q_{n}$ is

$$
\begin{aligned}
P\left(F Q_{n} ; \lambda\right)= & {[\lambda-(n-7)][\lambda-(n-3)]^{3} P\left(F Q_{n-1} ; \lambda-1\right) } \\
& \times \prod_{i=2}^{n-2} P\left(F Q_{n-i} ; \lambda-(i-4)\right) .
\end{aligned}
$$

Through calculating eigenvalues of characteristic polynomial and its multiplicities, we obtained that

(1) if $n \equiv 0(\bmod 2), F Q_{n}$ have $n / 2+1$ different eigenvalues $4 i-n+1$, with the multiplicities $C_{n}^{2 i}+C_{n}^{2 i+1}$, where $i=0,1, \ldots, n / 2$;
(2) if $n \equiv 1(\bmod 2), F Q_{n}$ have $(n+1) / 2$ different eigenvalues $4 i-n-1$, with the multiplicities $C_{n}^{2 i-1}+$ $C_{n}^{2 i}$, where $i=0,1, \ldots,(n+1) / 2$.

Combining with the definition of the Estrada index, we derived the result of Theorem 8 .

3.2. Some Bounds for the Estrada Index of Folded Hypercubes Networks $F Q_{n}$. It is well known that $F Q_{n}$ have $2^{n}$ vertices. Let $\lambda_{1} \geq \lambda_{2} \geq \cdots \geq \lambda_{n} \geq \lambda_{n+1} \geq \cdots \geq \lambda_{2^{n}}$ be the eigenvalues of $F Q_{n}$ with nonincreasing order. In order to obtain the bounds for the Estrada index of $F Q_{n}$, we prove some results by utilizing the arithmetic and geometric mean inequality; in our proof, some techniques in [22] are referred to.

Theorem 9. For any $F Q_{n}$ with $n \geq 2$, one has

$$
\begin{aligned}
& \sqrt{4^{n}+(n+1) 2^{n+1}+8 t+[\cosh (2)-3] M_{4}+\left[\cosh (2)-\frac{10}{3}\right] M_{5}} \\
& \quad<E E\left(F Q_{n}\right)
\end{aligned}
$$

where $M_{4}=2 Z g(G)-2 m+8 q, M_{5}=30 t+10 p+10 r, p$ and $q$ are the numbers of pentagons and quadrangles in $F Q_{n}$, and $r$ is the number of subgraphs consisting of a triangle with a pendent vertex attached.

Proof. In order to obtain the lower bounds for the Estrada index, consider that

$$
E E^{2}\left(F Q_{n}\right)=\sum_{i=1}^{2^{n}} e^{2 \lambda_{i}}+2 \sum_{i<j} e^{\lambda_{i}} e^{\lambda_{j}}
$$

Noting that $M_{0}=2^{n}, M_{1}=0, M_{2}=(n+1) 2^{n-1}$, and $M_{3}=6 t$, we obtain

$$
\begin{aligned}
\sum_{i=1}^{2^{n}} e^{2 \lambda_{i}}= & \sum_{i=1}^{2^{n}} \sum_{k \geq 0} \frac{\left(2 \lambda_{i}\right)^{k}}{k !} \\
= & 2^{n}+(n+1) 2^{n+1}+8 t+\sum_{i=1}^{2^{n}} \sum_{k \geq 4} \frac{\left(2 \lambda_{i}\right)^{k}}{k !} \\
= & 2^{n}+(n+1) 2^{n+1}+8 t+\sum_{k \geq 2} \frac{1}{(2 k) !} \sum_{i=1}^{2^{n}}\left(2 \lambda_{i}\right)^{2 k} \\
& +\sum_{k \geq 2} \frac{1}{(2 k+1) !} \sum_{i=1}^{2^{n}}\left(2 \lambda_{i}\right)^{2 k+1} .
\end{aligned}
$$

By Lemma 5,

$$
\sum_{i=1}^{n}\left(2 \lambda_{i}\right)^{k+2} \geq 4 \sum_{i=1}^{n}\left(2 \lambda_{i}\right)^{k},
$$


we can get that

$$
\begin{aligned}
\sum_{i=1}^{2^{n}} e^{2 \lambda_{i}} \geq & 2^{n}+(n+1) 2^{n+1}+8 t \\
& +\sum_{k \geq 2} \frac{1}{(2 k) !} \sum_{i=1}^{2^{n}} 2^{2 k-4}\left(2 \lambda_{i}\right)^{4} \\
& +\sum_{k \geq 2} \frac{1}{(2 k+1) !} \sum_{i=1}^{2^{n}} 2^{2 k-4}\left(2 \lambda_{i}\right)^{5} \\
= & 2^{n}+(n+1) 2^{n+1}+8 t+[\cosh (2)-3] M_{4} \\
& +\left[\cosh (2)-\frac{10}{3}\right] M_{5},
\end{aligned}
$$

where $M_{4}=2 Z g(G)-2 m+8 q, M_{5}=30 t+10 p+10 r, p$ and $q$ are the numbers of pentagons and quadrangles in $F Q_{n}$, and $r$ is the number of subgraphs consisting of a triangle with a pendent vertex attached.

As for the terms $2 \sum_{i<j} e^{\lambda_{i}} e^{\lambda_{j}}$, by the arithmetic and geometric mean inequality and the fact that $M_{1}=0$,

$$
\begin{aligned}
2 \sum_{i<j} e^{\lambda_{i}} e^{\lambda_{j}} & \geq 2^{n}\left(2^{n}-1\right)\left(\prod_{i<j} e^{\lambda_{i}} e^{\lambda_{j}}\right)^{2 / 2^{n}\left(2^{n}-1\right)} \\
& =2^{n}\left(2^{n}-1\right)\left[\left(\prod_{i=1} e^{\lambda_{i}}\right)^{2^{n}-1}\right]^{2 / 2^{n}\left(2^{n}-1\right)} \\
& =2^{n}\left(2^{n}-1\right)\left(e^{M_{1}}\right)^{2 / 2^{n}} \\
& =2^{n}\left(2^{n}-1\right)
\end{aligned}
$$

where the equality holds if and only if $\lambda_{1}=\cdots=\lambda_{2^{n}}$.

Combining with equalities (19) and (20),

$$
\begin{aligned}
& \sqrt{4^{n}+(n+1) 2^{n+1}+8 t+[\cosh (2)-3] M_{4}+\left[\cosh (2)-\frac{10}{3}\right] M_{5}} \\
& \quad \leq E E\left(F Q_{n}\right)
\end{aligned}
$$

where $M_{4}=2 Z g(G)-2 m+8 q, M_{5}=30 t+10 p+10 r, p$ and $q$ are the numbers of pentagons and quadrangles in $F Q_{n}$, and $r$ is the number of subgraphs consisting of a triangle with a pendent vertex attached.

Notice that the equality of (21) holds if and only if the equalities of (19) and (20) hold; that is, the equality holds if and only if $\lambda_{1}=\cdots=\lambda_{2^{n}}$, which is impossible for any $F Q_{n}$ with $n \geq 2$. Therefore, this implies the results of Theorem 9 .

We now consider the upper bound for the Estrada index of $F Q_{n}$ as follows.

Theorem 10. For any $F Q_{n}$ with $n \geq 2$, one has

$$
E E\left(F Q_{n}\right)<2^{n}-1+e^{\sqrt{(n+1) 2^{n}}} .
$$

Proof. According to the definition of Estrada index we get

$$
\begin{aligned}
E E\left(F Q_{n}\right) & =2^{n}+\sum_{i=1}^{2^{n}} \sum_{k \geq 1} \frac{\lambda_{i}^{k}}{k !} \leq 2^{n}+\sum_{i=1}^{2^{n}} \sum_{k \geq 1} \frac{\left|\lambda_{i}\right|^{k}}{k !} \\
& =2^{n}+\sum_{k \geq 1} \frac{1}{k !} \sum_{i=1}^{2^{n}}\left[\left(\lambda_{i}\right)^{2}\right]^{k / 2} .
\end{aligned}
$$

Notice the inequality

$$
\sum_{i=1}^{2^{n}}\left[\left(\lambda_{i}\right)^{2}\right]^{k / 2} \leq\left[\sum_{i=1}^{2^{n}}\left(\lambda_{i}\right)^{2}\right]^{k / 2}
$$

substituting inequality (24) into (23) we obtain that

$$
\begin{aligned}
E E\left(F Q_{n}\right) & \leq 2^{n}+\sum_{k \geq 1} \frac{1}{k !}\left[\sum_{i=1}^{2^{n}}\left(\lambda_{i}\right)^{2}\right]^{k / 2} \\
& =2^{n}-1+\sum_{k \geq 0} \frac{1}{k !}\left[\sum_{i=1}^{2^{n}}\left(\lambda_{i}\right)^{2}\right]^{k / 2} .
\end{aligned}
$$

Since the equality holds in $F Q_{n}$,

$$
\sum_{i=1}^{2^{n}}\left(\lambda_{i}\right)^{2}=(n+1) 2^{n}
$$

Hence,

$$
\begin{aligned}
E E\left(F Q_{n}\right) & \leq 2^{n}-1+\sum_{k \geq 0} \frac{1}{k !}\left[(n+1) 2^{n}\right]^{k / 2} \\
& =2^{n}-1+\sum_{k \geq 0} \frac{\sqrt{(n+1) 2^{n}}}{k !} \\
& =2^{n}-1+e^{\sqrt{(n+1) 2^{n}}} .
\end{aligned}
$$

It is evident that equality of (25) will be attained if and only if the graph $F Q_{n}$ has no nonzero eigenvalues, which, in turn, happens only in the case of the edgeless graph $\overline{K_{n}}$; it is impossible for any $F Q_{n}$ with $n \geq 2$ that directly leads to the inequality in (27).

Hence, we can obtain the upper bound for the Estrada index of $F Q_{n}$ :

$$
E E\left(F Q_{n}\right)<2^{n}-1+e^{\sqrt{(n+1) 2^{n}}} .
$$

The proof of Theorem 10 is completed.

Remark 11. In [23], it was proved that

$$
e^{r}+(n-1) e^{-r /(n-1)} \leq E E(G)<n-2+e^{r}+e^{\sqrt{r(n-r)-1}},
$$

with equality, holds if and only if $\lambda_{2}=\lambda_{3}=\cdots=\lambda_{n}=$ $-r /(n-1)$. 
Notice that the spectral radius of $F Q_{n}$ is $\lambda_{1}=n+1$ and $r=n+1$; applying Lemma 6 , we also give the lower and upper bounds connecting $E E\left(F Q_{n}\right)$ and its spectral radius by simple computations, where the equality is impossible for any $F Q_{n}$; hence

$$
\begin{aligned}
e^{n+1}+ & \left(2^{n}-1\right) e^{(-n-1) /\left(2^{n}-1\right)} \\
& <E E\left(F Q_{n}\right)<2^{n}-2+e^{n+1}+e^{\sqrt{(n+1)\left[2^{n}-(n+1)\right]-1}} .
\end{aligned}
$$

3.3. Some Properties on Estrada Index Involving Energy of $F Q_{n}$. In this section, we investigate the relations between the Estrada index and the energy of $F Q_{n}$. We firstly prove the lower bounds involving energy for the Estrada index of $F Q_{n}$; in Theorem 12 proof, some techniques in [23] are referred to.

Theorem 12. For any $F Q_{n}$ with $n \geq 2$, one has

$$
\frac{1}{2}(e-1) E\left(F Q_{n}\right)+\left(2^{n}-n_{i}\right)<E E\left(F Q_{n}\right) .
$$

Proof. Assume that $n_{i}$ denote the number of positive eigenvalues; we begin with the definition of Estrada index $E E\left(F Q_{n}\right)$ :

$$
E E\left(F Q_{n}\right)=\sum_{i=1}^{2^{n}} e_{i}^{\lambda}=\sum_{\lambda_{i} \leq 0} e_{i}^{\lambda}+\sum_{\lambda_{i}>0} e_{i}^{\lambda} .
$$

Since $e^{x} \geq 1+x$, with equality, holds if and only if $x=0$, we have

$$
\sum_{\lambda_{i} \leq 0} e_{i}^{\lambda} \geq \sum_{\lambda_{i} \leq 0}\left(1+\lambda_{i}\right)=\left(2^{n}-n_{i}\right)+\left(\lambda_{n_{i}+1}+\cdots+\lambda_{n}\right) .
$$

The other underlying inequality is $e^{x} \geq e x$ and equality holds if and only if $x=1$; we get

$$
\sum_{\lambda_{i}>0} e_{i}^{\lambda} \geq \sum_{\lambda_{i}>0} e \lambda_{i}=e\left(\lambda_{1}+\lambda_{2} \cdots+\lambda_{n_{i}}\right) .
$$

Substituting the inequalities (33) and (34) into (32),

$$
\begin{aligned}
E E\left(F Q_{n}\right) \geq & \left(2^{n}-n_{i}\right)+\left(\lambda_{n_{i}+1}+\cdots+\lambda_{n}\right) \\
& +e\left(\lambda_{1}+\lambda_{2} \cdots+\lambda_{n_{i}}\right) \\
= & \left(2^{n}-n_{i}\right)+\left(\lambda_{1}+\lambda_{2} \cdots+\lambda_{n_{i}}+\lambda_{n_{i}+1}+\cdots+\lambda_{n}\right) \\
& +(e-1)\left(\lambda_{1}+\lambda_{2} \cdots+\lambda_{n_{i}}\right) \\
= & \left(2^{n}-n_{i}\right)+(e-1)\left(\lambda_{1}+\lambda_{2} \cdots+\lambda_{n_{i}}\right) .
\end{aligned}
$$

Note that

$$
\lambda_{1}+\lambda_{2} \cdots+\lambda_{n_{i}}=\frac{1}{2} E\left(F Q_{n}\right) .
$$

From the above inequalities (35) and (36), we arrive at

$$
\frac{1}{2}(e-1) E\left(F Q_{n}\right)+\left(2^{n}-n_{i}\right) \leq E E\left(F Q_{n}\right),
$$

with equality if and only if $F Q_{n}$ is an empty graph with $2^{n}$ vertices, which is impossible.

Hence,

$$
\frac{1}{2}(e-1) E\left(F Q_{n}\right)+\left(2^{n}-n_{i}\right)<E E\left(F Q_{n}\right),
$$

as desired.

We now derive the upper bounds involving energy for the Estrada index of $F Q_{n}$.

Theorem 13. For any $F Q_{n}$ with $n \geq 2$, one has

$$
E E\left(F Q_{n}\right)<E\left(F Q_{n}\right)+2^{n}-1-\sqrt{(n+1) 2^{n}}+e^{\sqrt{(n+1) 2^{n}}} .
$$

Proof. We consider that

$$
\begin{aligned}
E E\left(F Q_{n}\right) & =\sum_{i=1}^{n} e_{i}^{\lambda}=2^{n}+\sum_{i=1}^{2^{n}} \sum_{k \geq 1} \frac{\lambda_{i}^{k}}{k !} \\
& \leq 2^{n}+\sum_{i=1}^{2^{n}} \sum_{k \geq 1} \frac{\left|\lambda_{i}\right|^{k}}{k !} .
\end{aligned}
$$

Taking into account the definition of graph energy equation (1), we obtain

$$
\begin{aligned}
E E\left(F Q_{n}\right) & \leq 2^{n}+E\left(F Q_{n}\right)+\sum_{i=1}^{2^{n}} \sum_{k \geq 2} \frac{\left|\lambda_{i}\right|^{k}}{k !} \\
& =2^{n}+E\left(F Q_{n}\right)+\sum_{k \geq 2} \frac{1}{k !} \sum_{i=1}^{2^{n}}\left[\left(\lambda_{i}\right)^{2}\right]^{k / 2} .
\end{aligned}
$$

In light of the inequality (24) holds for integer $k \geq 2$, we obtain that

$$
\begin{aligned}
E E\left(F Q_{n}\right) \leq & 2^{n}+E\left(F Q_{n}\right)+\sum_{k \geq 2} \frac{1}{k !}\left[\sum_{i=1}^{2^{n}}\left(\lambda_{i}\right)^{2}\right]^{k / 2} \\
= & 2^{n}+E\left(F Q_{n}\right)-1-\sqrt{(n+1) 2^{n}} \\
& +\sum_{k \geq 0} \frac{1}{k !}\left[\sum_{i=1}^{2^{n}}\left(\lambda_{i}\right)^{2}\right]^{k / 2} .
\end{aligned}
$$

Substituting (26) into (42), we get

$$
\begin{aligned}
E E\left(F Q_{n}\right) \leq & 2^{n}+E\left(F Q_{n}\right)-1-\sqrt{(n+1) 2^{n}} \\
& +\sum_{k \geq 0} \frac{1}{k !}\left[(n+1) 2^{n}\right]^{k / 2} \\
= & 2^{n}+E\left(F Q_{n}\right)-1-\sqrt{(n+1) 2^{n}}+e^{\sqrt{(n+1) 2^{n}}},
\end{aligned}
$$

with equality if and only if $F Q_{n}$ is an empty graph with $2^{n}$ vertices, which is impossible.

From the above argument, we get the result of Theorem 13. 


\section{Conclusions}

The main purpose of this paper is to investigate the Estrada index of $F Q_{n}$ with $n \geq 2$; we established the explicit formulae for calculating the Estrada index of $F Q_{n}$ by deducing the characteristic polynomial of the adjacency matrix in spectral graph theory.

Moreover, some lower and upper bounds for Estrada index of $F Q_{n}$ were proposed by utilizing the arithmetic and geometric mean inequality. The lower and upper bounds for the Estrada index involving energy of $F Q_{n}$ were also obtained.

\section{Conflict of Interests}

The authors declare that there is no conflict of interests regarding the publication of this paper.

\section{Acknowledgments}

The work of Jia Bao Liu was supported by the Natural Science Foundation of Anhui Province of China under Grant no. KJ2013B105; the work of Xiang-Feng Pan was supported by the National Science Foundation of China under Grants 10901001, 11171097, and 11371028.

\section{References}

[1] J. M. Xu, Topological Strucure and Analysis of Interconnction Networks, Kluwer Academic, Dordrecht, The Netherlands, 2001.

[2] I. Gutman, “The energy of a graph," Berichte der MathematischStatistischen Sektion im Forschungszentrum Graz, vol. 103, no. 22, pp. 100-105, 1978.

[3] E. Estrada, "Characterization of 3D molecular structure," Chemical Physics Letters, vol. 319, no. 5, pp. 713-718, 2000.

[4] E. Estrada and J. A. Rodríguez-Velázquez, "Subgraph centrality in complex networks," Physical Review E, vol. 71, no. 5, Article ID 056103, 9 pages, 2005.

[5] E. Estrada and J. A. Rodríguez-Velázquez, "Spectral measures of bipartivity in complex networks," Physical Review E, vol. 72, no. 4, Article ID 046105, 6 pages, 2005.

[6] K. C. Das, I. Gutman, and B. Zhou, "New upper bounds on Zagreb indices," Journal of Mathematical Chemistry, vol. 46, no. 2, pp. 514-521, 2009.

[7] D. M. Cvetković, M. Doob, I. Gutman, and A. Torgašev, Recent Results in the Theory of Graph Spectra, vol. 36 of Annals of Discrete Mathematics, North-Holland, Amsterdam, The Netherlands, 1988.

[8] E. A. Amawy and S. Latifi, "Properties and performance of folded hypercubes," IEEE Transactions on Parallel and Distributed Systems, vol. 2, no. 1, pp. 31-42, 1991.

[9] R. Indhumathi and S. A. Choudum, "Embedding certain height-balanced trees and complete $p^{m}$-ary trees into hypercubes," Journal of Discrete Algorithms, vol. 22, pp. 53-65, 2013.

[10] J. Fink, "Perfect matchings extend to Hamilton cycles in hypercubes," Journal of Combinatorial Theory B, vol. 97, no. 6 , pp. 1074-1076, 2007.

[11] J. B. Liu, J. D. Cao, X. F. Pan, and A. Elaiw, "The Kirchhoff index of hypercubes and related complex networks," Discrete Dynamics in Nature and Society, vol. 2013, Article ID 543189, 7 pages, 2013.
[12] M. Chen and B. X. Chen, "Spectra of folded hypercubes," Journal of East China Normal University (Nature Science), vol. 2, pp. 3946, 2011.

[13] X. He, H. Liu, and Q. Liu, "Cycle embedding in faulty folded hypercube," International Journal of Applied Mathematics \& Statistics, vol. 37, no. 7, pp. 97-109, 2013.

[14] S. Cao, H. Liu, and X. He, "On constraint fault-free cycles in folded hypercube," International Journal of Applied Mathematics \& Statistics, vol. 42, no. 12, pp. 38-44, 2013.

[15] Y. Zhang, H. Liu, and M. Liu, "Cycles embedding on folded hypercubes with vertex faults," International Journal of Applied Mathematics \& Statistics, vol. 41, no. 11, pp. 58-70, 2013.

[16] X. B. Chen, "Construction of optimal independent spanning trees on folded hypercubes," Information Sciences, vol. 253, pp. 147-156, 2013.

[17] M. Chen, X. Guo, and S. Zhai, "Total chromatic number of folded hypercubes," Ars Combinatoria, vol. 111, pp. 265-272, 2013.

[18] G. H. Wen, Z. S. Duan, W. W. Yu, and G. R. Chen, "Consensus in multi-agent systems with communication constraints," International Journal of Robust and Nonlinear Control, vol. 22, no. 2, pp. 170-182, 2012.

[19] J. B. Liu, X. F. Pan, Y. Wang, and J. D. Cao, “The Kirchhoff index of folded hypercubes and some variant networks," Mathematical Problems in Engineering, vol. 2014, Article ID 380874, 9 pages, 2014.

[20] E. Estrada, J. A. Rodríguez-Velázquez, and M. Randić, "Atomic branching in molecules," International Journal of Quantum Chemistry, vol. 106, no. 4, pp. 823-832, 2006.

[21] B. Zhou and Z. Du, "Some lower bounds for Estrada index," Iranian Journal of Mathematical Chemistry, vol. 1, no. 2, pp. 6772, 2010.

[22] Y. Shang, "Lower bounds for the Estrada index of graphs," Electronic Journal of Linear Algebra, vol. 23, pp. 664-668, 2012.

[23] J. P. Liu and B. L. Liu, "Bounds of the Estrada index of graphs," Applied Mathematics, vol. 25, no. 3, pp. 325-330, 2010. 


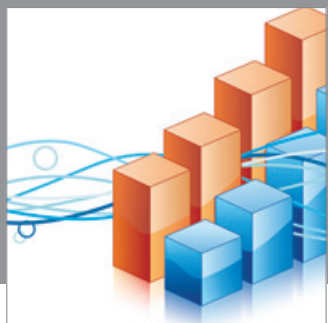

Advances in

Operations Research

mansans

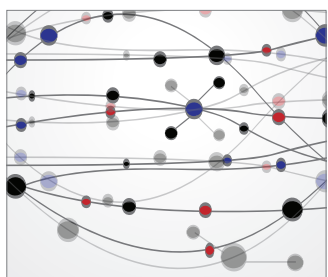

The Scientific World Journal
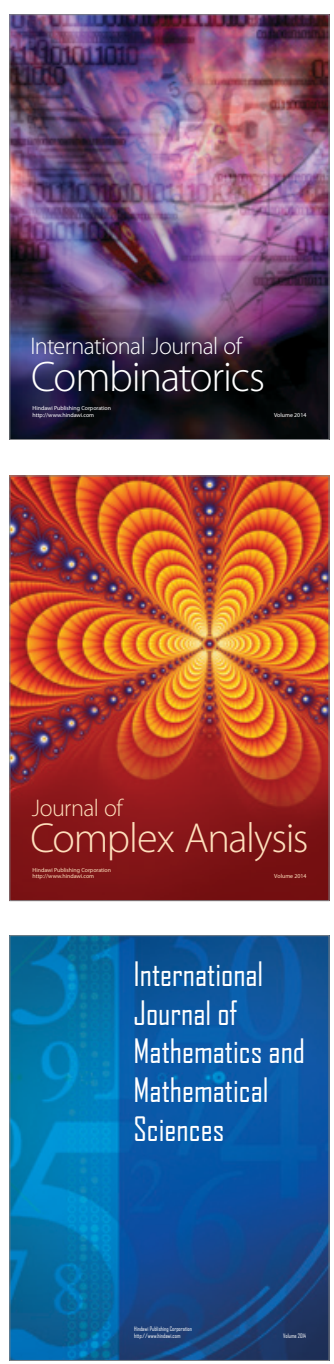
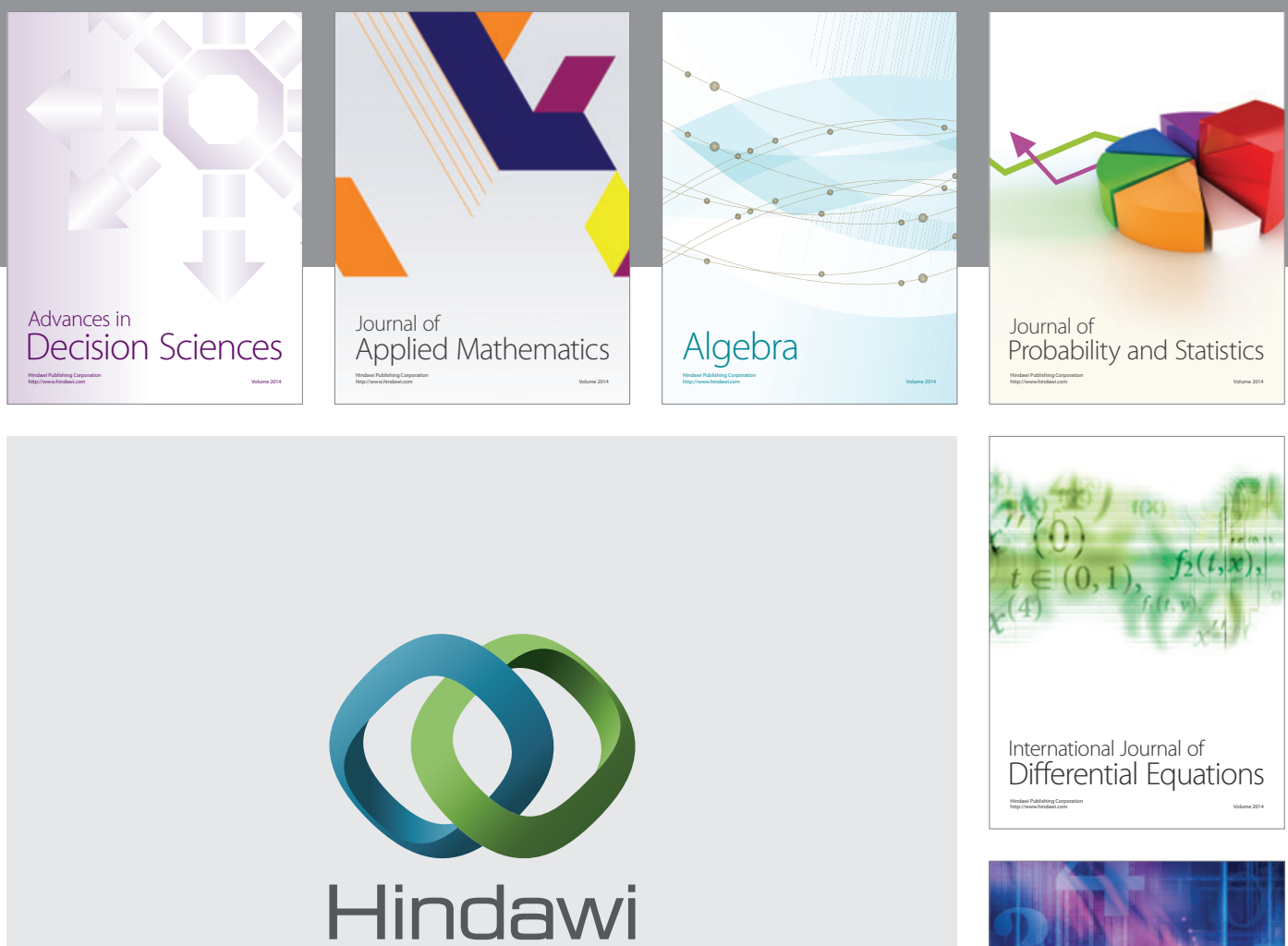

Submit your manuscripts at http://www.hindawi.com
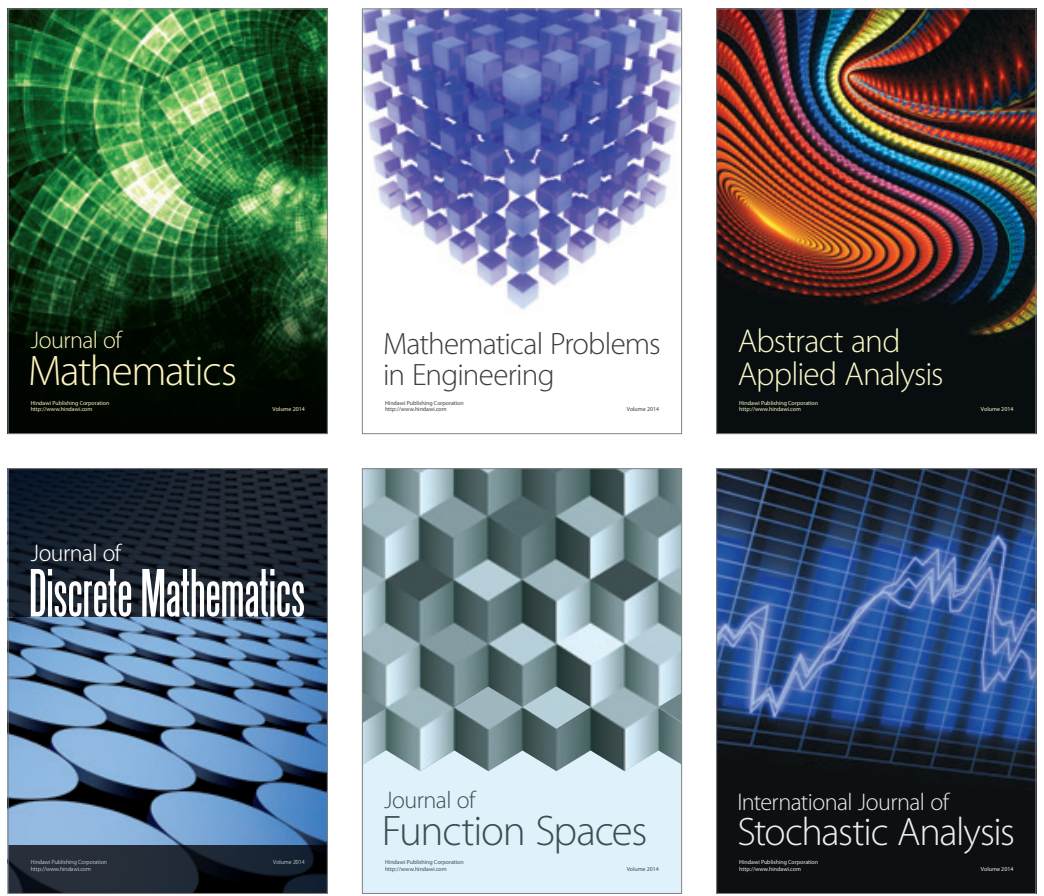

Journal of

Function Spaces

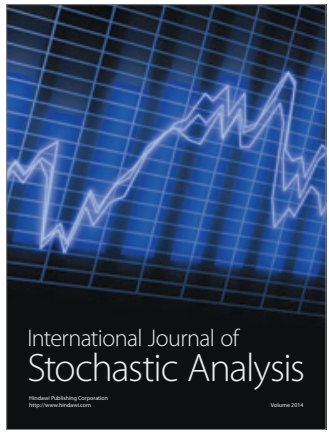

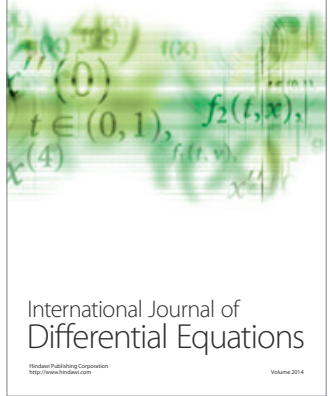
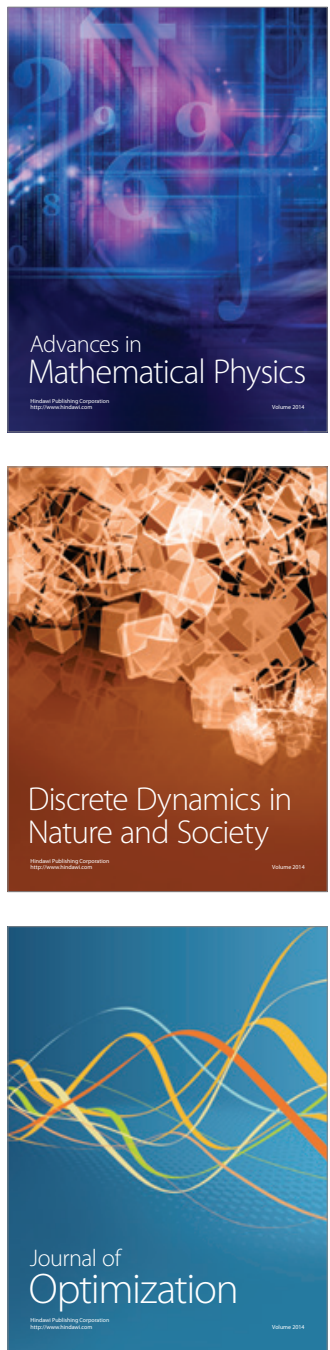